\title{
Cooperative Robot Structures Modeled After Whale Behavior and Social Structure
}

\author{
I.C. Reșceanu, G.C. Călugăru, C.F. Reșceanu, N.G. Bîzdoacă
}

\author{
Ionut Cristian Resceanu \\ Cristina Floriana Resceeanu \\ Nicu-George Bîzdoacă \\ University of Craiova \\ Faculty of Automation, Computers and Electronics \\ Romania, 200440 Craiova, Decebal Blvd., no. 107 \\ E-mail: $\{$ resceanu,cristina,nicu\}@robotics.ucv.ro

\section{George-Cristian Călugăru} \\ University of Craiova \\ Faculty of Automation, Computers and Electronics \\ Romania, 200440 Craiova, Decebal Blvd., no. 107 \\ E-mail: calugaru.george.nds@gmail.ro
}

\begin{abstract}
:
This paper analyses the communications and social structure of whale pods and tries to apply their principles on cooperative robot structures which can be guided to perform a certain task. The communication patterns and social structure are presented at first in order to define real, natural phenomena which can then be translated through cooperative robotic structures. Whale communication has suffered modifications in the latter years mostly because of the increase of noise in the ocean. Problems regarding communications between the cooperative structures chosen for modelling whale behavior are solved by data fusion techniques. In the last part of the paper the problem of dynamic compensation of disturbances is studied with regard to cooperative structures.
\end{abstract}

Keywords: cooperative structures, data fusion, manipulative robots.

\section{Introduction}

The order Cetacea (Cetus, whale, from Greek) includes the marine mammals commonly known as whales, dolphins and porpoises. Cetus is Latin and is used in biological names to mean "whale"; its original meaning, "large sea animal", was more general. It comes from Ancient Greek (ketos), meaning "whale" or "any huge fish or sea monster". In Greek mythology the monster Perseus defeated was called Ceto, which is depicted by the constellation of Cetus. Cetology is the branch of marine science associated with the study of cetaceans. Suborders of the Cetacea order are Mysticeti, Odontoceti and Archaeoceti (extinct ancient whales, depict the evolution of whales throughout time). Cetaceans are mammals that evolved throughout the ages for aquatic environments. Their body is fusiform (spindle-shaped) and the forelimbs are modified into flippers as a result of this evolution. The tiny hindlimbs are vestigial; they do not attach to the backbone and are hidden within the body. The tail has horizontal flukes. Cetaceans are nearly hairless, and are insulated from the cooler water they inhabit by a thick layer of blubber. Some species are noted for their high intelligence.

\subsection{Whale Social Structure}

Whale family structures are fascinating. One of the most important facts about whales is that they are particularly intelligent mammals and like humans, place much value on their families 
and the role that each member plays within the unit. Notably, the individual families also travel and migrate together in pods. Each family member continues to play a vital role within that pod, as a greater unit of the family. These groups demonstrate the sociable nature of whales and their unspoken cooperation with one another is evidence of the insight and sense of responsibility inherent to these animals.

Another interesting whale fact: whales tend to separate themselves into pods according to age and sex. The whale cows and their calves travel together in pods of up to 30 members at a time, accompanied by one dominant bull. Cows without their calves, or whose calves are mature enough, act as midwives to pregnant and nursing mothers. They assist them with their birth by ensuring that the newborn reaches the surface of the water for air. Cows are also babysitters to the other mother's calves in her absence, and assist her with the care of her new baby in a general sense.

The calves stick close to their mothers for an average of three to six years. But this period can be even longer, depending on the individual calf and their species. Even after they have left their mother's side, they may still return to the main pod to visit her. Females are also known for returning to their first pod when they become pregnant with their own calf.

The youths of the group eventually branch off into a smaller juvenile pod. They will move into larger pods once they reach sexual maturity and begin to calve. In some cases, the juvenile pod is either replaced or broken away from by a 'bachelor pod', consisting of only the young bulls. Whales are considered 'juveniles' from about three years of age to approximately thirteen.

There is a dominant bull in each core pod, and he is responsible for the pod in which he resides. He is sexually mature and cares for his harem of cows and calves. The other males tend to stick to themselves, traveling separately from the rest of the main pod, as to respect the 'property' of the dominant bull. This family- and pod structure is designed to protect the weak and the young of the group. Because whale calves do not mature as quickly as some other mammals do, they require time to grow and develop within a protected environment. The organization of the dominant male and the group of mothering cows ensures that calves are isolated from the dangers of the deep.

Traveling in this way also ensures that whale migrations remain orderly and safe for all involved, preventing smaller family units from drifting off course or facing the dangers that come with isolation. Of course, whales are also social creatures and benefit from the close interaction with others of their sort. This level of mutual understanding and cooperation is another indication of the brilliance of the whale creation and instinct. [12]

\subsection{Whale Communication}

Whales can communicate through a very intriguing method called echolocation. Echolocation, also known as biosonar, is the biological sonar used by several animals such as shrews, most bats and most cetaceans. The term was first used by Donald Griffin, whose work with Robert Galambos was the first to conclusively demonstrate its existence in bats. Two bird groups also employ this system for navigating through caves, the so called cave swiftlets in the genus Aerodramus (formerly Collocalia) and the unrelated Oilbird Steatornis caripensis. [13]

Echolocating animals emit calls out to the environment and listen to the echoes of those calls that return from various objects in the environment. They use these echoes to locate, range and identify the objects. Echolocation is used for navigation and for foraging (or hunting) in various environments.

Echolocation makes use of active sonar, using sounds made by an animal. Ranging is done by measuring the time delay between the animal's own sound emission and any echoes that return from the environment. The relative intensity of sound received at each ear provides information 
about the horizontal angle (azimuth) from which the reflected sound waves arrive. Unlike some sonar that relies on an extremely narrow beam to localize a target, animal echolocation relies on multiple receivers. Echolocating animals have two ears positioned slightly apart. The echoes returning to the two ears arrive at different times and at different loudness levels, depending on the position of the object generating the echoes. The time and loudness differences are used by the animals to perceive distance and direction. With echolocation, the bat or other animal can see not only where it is going but also how big another animal is, what kind of animal it is, and other features.

The sounds produced by whales can travel for miles as objects found in the water can help amplify them. The sounds will echo back to the whale that emitted them. This form of communication has an estimated speed of 1 mile/second.

Sounds made by whales are in fact very unique. The clicks are a basic part of this communication. The clicks also help the whales navigate through the waters as well as being a language feature. Different species of whales communicate in various ways. This is due to the fact that they don't have an inner ear.

The humpback whales have a very particular way of communication called singing. This signing mainly consists of periodical sound patterns. These songs can be up to 30 minutes in length and can travel up to 100 miles. Sperm whales have only been heard making clicks, while toothed whales use echolocation that can generate sounds of 30000 Watts at 163 decibels. Whales create small pods with various communication patterns within. Researchers established that whales from different locations in the world have different terminology. This terminology is also different at whales in captivity in comparison with free whales.

It is believed that whales have amplified their songs in the past few decades. Whale songs can travel thousands of miles, but new research shows that their ability to communicate is severely affected by the increase of the amount of noise in an ocean. Thus the whales adapted by making their songs louder. [11]

\section{Using Data Fusion for Process Surveillance and Diagnosis In the Modelling of Whale Communication}

Terms like data fusion, multi-sensor data fusion, sensor fusion and information fusion or multi-sensor integration are used frequently in literature to depict a variety of techniques, technologies, systems and applications which use data provided by multiple sources. The data fusion applications are various - from real-time sensor fusion applied to mobile robots navigation to the fusion of strategic intelligence developed for military purposes. [6]

Data fusion is used in the development of modern applications regarding process surveillance and diagnosis.

Using specific algorithms, damaged sensors can be determined and in the same time signals can be synthesized in order to replace the erroneous information.

Data fusion techniques combine data from a multitude of sensors and related information to obtain more specific results than in the case of one sensor.

The data fusion concept can find its equivalent in nature. Throughout their evolution, people and animals developed their ability to use multiple senses in order to survive. For example, establishing if a food is eatable or not cannot be determined by using only the sight sense; the combination of sight, touch, smell and taste is far more effective. In a similar manner, when the ability to see is blocked by built structures or vegetation, the hearing sense can offer advanced warnings in case of imminent danger. Thus, multi-sensor data fusion is performed naturally by animals and people in order to evaluate the configuration of the surrounding environment and 
in order to detect possible threats.

Even though the concept of data fusion is well established, the development of new sensors, new techniques of processing and hardware made real-time data fusion viable.

Recent progresses made in the development of computers and sensors offer the ability to emulate the natural abilities regarding data fusion for both people and animals through hardware and software means. Nowadays, data fusion systems are used in military applications such as: target recognition (e.g. intelligent weapons), vehicle guidance and remote detection like IFFN systems (Identification Friend-Foe-Neutral Systems). Non-military applications include monitoring of manufacturing processes, condition based maintenance for complex machinery, robotics and medical applications.

\subsection{Multi-sensor Advantages}

Another frequent term is that of multi-sensor integration which signifies the synergic use of sensor data to perform a specific task.

Sensor fusion is different from multi-sensor integration because the first includes the current combination of sensor information in a single representation format.

Multiple sensor data fusion offers multiple advantages in comparison with information prelevated through one sensor. First, if multiple sensors are used (e.g. identical radars following an object in motion) combining all the determinations will provide a better estimate of the motion and position of the object. A statistical advantage is obtained by adding $\mathrm{N}$ independent determinations (e.g. estimation of the position and speed of the target is improved by a factor of ), with the assumption that data are combined in an optimal manner. The same result could be obtained by adding $\mathrm{N}$ independent determinations to a single sensor.

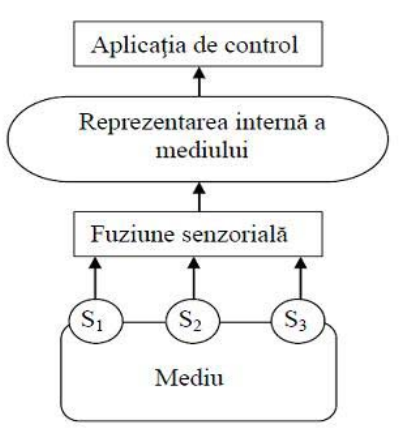

a) Fuziune senzorială

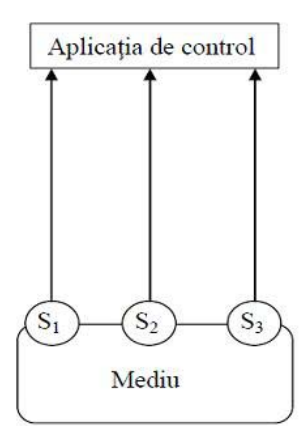

b) Integrare multisenzorială

Figure 1: Data fusion and multiple sensor integration configurations

A second advantage implies the use of relative placement and motion of sensors to improve the process of observation. For example, 2 sensors that measure the angular motion of an object can be coordinated in order to determine the position of an object through triangularization. This technique is used for surveillance and commercial navigation.

A third advantage is the improved observability. The initial increase in physical observability may lead to significant improvements.

\subsection{Control Architectures}

For system control, the following types of data fusion can be defined with regard to sensors, [1]:

- Complementary data fusion - the fusion of multiple sensors spread across an area provides partial information about the environment; e.g. the fusion of multiple sensors which mea- 
sure distances or video cameras pointing in different directions. This type of fusion solves the problem of incomplete information.

- Competitive fusion - the fusion of uncertain data obtained from multiple sensors - e.g. a radar and a video camera which detect the same object. Through data fusion, the distance to the object can be obtained with a higher accuracy. This type of fusion is used to reduce the effect of uncertainties and erroneous measurements.

- Cooperative fusion - the fusion of different sensors in which one of the sensors is based on determinations of another sensor in order to obtain its own set of data. For example, when a tactile sensor supplies information about the shape of an object previously estimated by a proximity sensor. This type of fusion is used to diminish the uncertainty effect, measurement errors as well as the incomplete state of the data.

Three basic alternatives can be used for multi-sensor data:

1. Direct fusion of data provided by sensors

2. Representation of sensor data through characteristic vectors with subsequent fusions of them.

3. Processing of each sensor in order to obtain high level control decisions which are later combined. Each of these approaches will use different data fusion techniques.

If multi-sensor data are proportional (if sensors measure the same physical phenomenon) then data from the sensors can be directly combined. The techniques for fusion of sheer data imply classical estimation methods like Kalman filtering. However, if sensor data is not proportional the data must be merged at the level of the characteristic/state vector or at a decision level.

Data fusion at the characteristics level implies the extraction of the representative characteristics from the sensor. It has been demonstrated that people use a cognitive function based on characteristics in order to determine objects. In case of data fusion at the characteristics level, the characteristics are extracted from multiple sensor observers and combined in a single vector which is subjected to pattern recognition techniques like neural networks or grouping algorithms.

Decision level data fusion combines sensor information after every sensor performed a preliminary determination of the entity location, attributes and identification.

\subsection{A Model for Processing Data Fusion}

In order to improve communications between military researchers and system developers, JDL (Joint Directors of Laboratories), established in 1986, began an effort to define the specific terms regarding data fusion. The result of the effort led to the creation of a process model for data fusion and specific terminology. The JDL process model is set to be very general and useful in multiple application domains, identifies processes and categories of techniques applicable to data fusion. The model is hierarchical with 2 levels. At the upper level, the data fusion process is described by the sensor inputs, the interaction between man and computer, database management, source preprocessing and 4 key sub-processes:

Level 1 processing (object refining) combines sensor data to obtain the most reliable and accurate estimation of the position of the object, its velocity, attributes and identity.

Level 2 processing (scenario refining) tries dynamically to develop a description of the current relationship between entities and events taking place in their surrounding environment.

Level 3 processing (threat refining) projects the current scenario into the future in order to draw the inferences about for threats, about friends and the foe vulnerabilities and opportunities of action. 

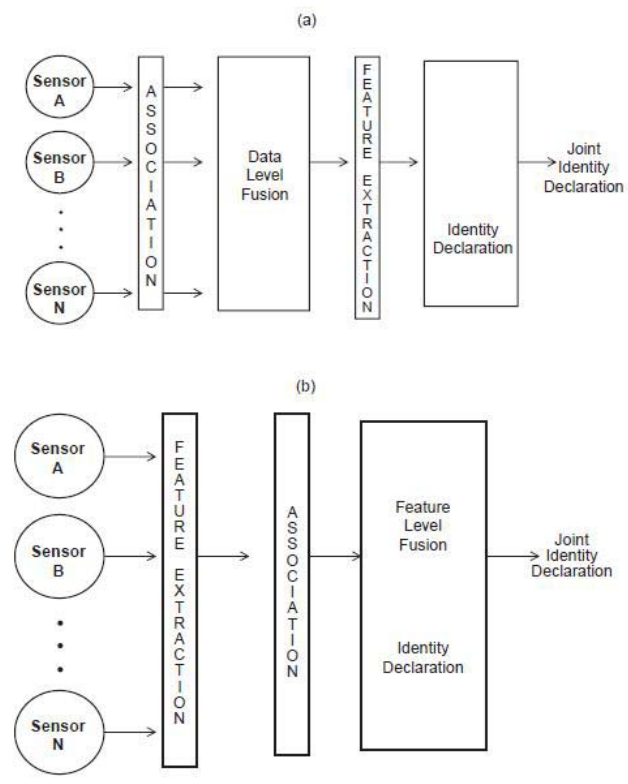

(c)

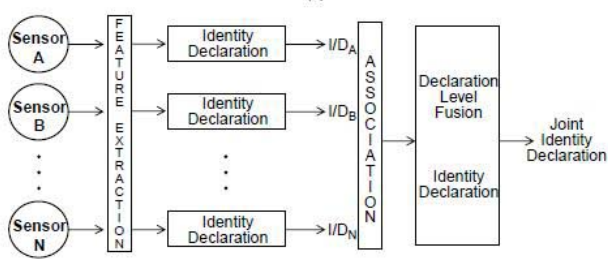

Figure 2: a. Direct data fusion of sensors; b. Representation of sensor data through characteristic vectors and subsequent fusions of them; c. The processing of each sensor in order to obtain high level inferences and decisions which are then combined

Level 4 processing (process refining) is a meta-process which monitors the global process of data fusion in order to evaluate and improve the real-time system.

For each of these sub-processes, the JDL hierarchical model identifies specific function and technique categories (in the second level of the model) and specific techniques (in the lower layer of the model). The implementation of the data fusion systems integrates and correlates those functions in a general work flow.

\section{The Dynamic Model of a Manipulative Robot Used In Mod- elling Whale Behavior}

The cooperative structure used to model whale behavior contains manipulative robots. The dynamic model of a such a robot is presented below. For more details on the principles of bioinsipired computing and robots see [10]. Also for more details on cooperative robot structures see [3].

The dynamic model is given by:

$$
\begin{aligned}
& J \prime_{1} \ddot{\theta}_{1}+J^{*} \cos \left(\theta_{1}-\theta_{2}\right) \ddot{\theta}_{2}+J^{*} \sin \left(\theta_{1}-\theta_{2}\right) \dot{\theta}_{2}^{2}+M \prime_{1} \cos \theta_{1}=M_{1} \\
& J \prime_{2} \ddot{\theta}_{2}+J^{*} \cos \left(\theta_{1}-\theta_{2}\right) \ddot{\theta}_{1}-J^{*} \sin \left(\theta_{1}-\theta_{2}\right) \dot{\theta}_{1}^{2}+M \prime_{2} \cos \theta_{2}=M_{2}
\end{aligned}
$$




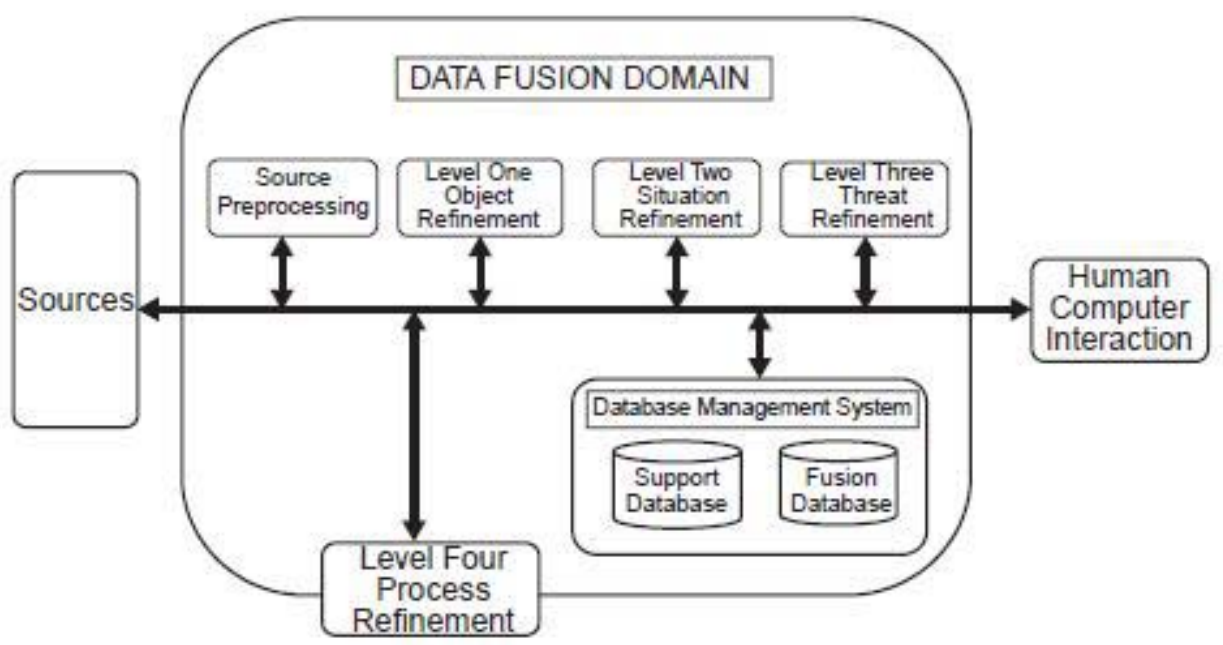

Figure 3: The JDL process model for data fusion

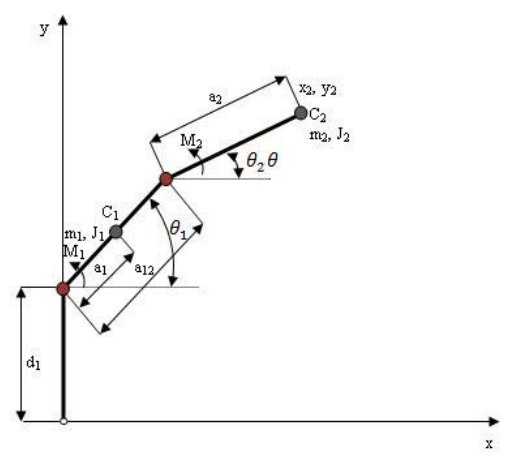

Figure 4: The kinematical model of the manipulative robot

\subsection{Determining the Operation Span In Fault Conditions}

The present day migration courses of whale pods are affected by naval traffic. For instance, in 2007 a mother whale and her calf were trying to return from the Sacramento- San Joaquin Delta to the Pacific Ocean. The two whales had deviated off course a week earlier. The change in course is believed to be influenced by the sounds of tug boats met along the way. The two whales appeared to have been wounded by a ship's propeller. In case the whale swimmers are affected, the whale will try to maintain its course using the wounded swimmers with a smaller operation span. In this paragraph the operation span in fault conditions is determined.

According to the kinematical configuration of the robot system considered, it has an area of operation in the shape of an annulus sector as depicted in the figure below:

In the case of a fabrication line which contains a number of robots with the same kinematical configuration, trespassing over these admissible operation areas can lead to interference problems. In order to avoid this, all areas that can be trespassed are eliminated apriori and thus each robot can have its own distinct area. So, for every robot a rectangular operation area (called an operation cell) will be defined.

Fig. 5.a) depicts the three parameters that describe the area of operation: maximum angle $\varphi$, minimum range $r_{\min }$ and respectively, the maximum range $r_{\max }$.

In figure 6.b) a rectangular operation cell is defined where $\mathrm{x}$ and $\mathrm{y}$ represent the height and width of the cell. Between the 2 regions the following relations are defined: 


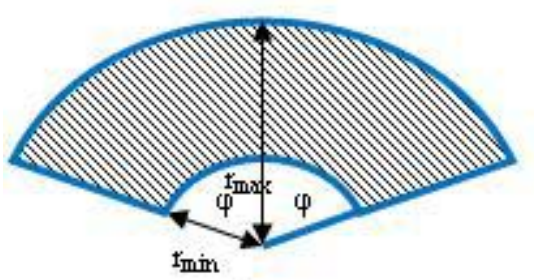

a)

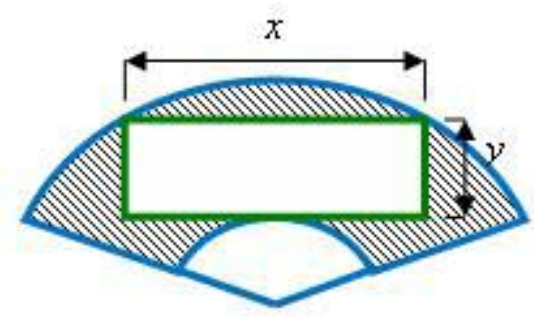

b)

Figure 5: Definition of the area of operation for the manipulative robot: a) area of operation b) definition of the operation cell

$$
\begin{gathered}
\frac{y}{2}<r_{\text {min }} t g \varphi \\
r_{\text {max }}^{2}=\left(r_{\text {min }}+y\right)^{2}+\left(\frac{y}{2}\right)^{2}
\end{gathered}
$$

\subsection{Manipulator Fault Due to a Jammed Articulation}

It is assumed that the fault which appears at the manipulator is due to a jammed articulation. It is also assumed that the value of the angle of the jammed articulation is known. Even though the blocking position is not available from the sensor attached to every articulation, this can be calculated based on the position of the griper of the faulted manipulator by solving backward kinematics problems.

An Articulation Fault

When the first articulation is jammed, the faulted manipulator can only move its third joint by means of the second joint.

Depending on the configuration of the system at the moment the fault occurred, the faulted manipulator can be placed in a position similar to the one below:

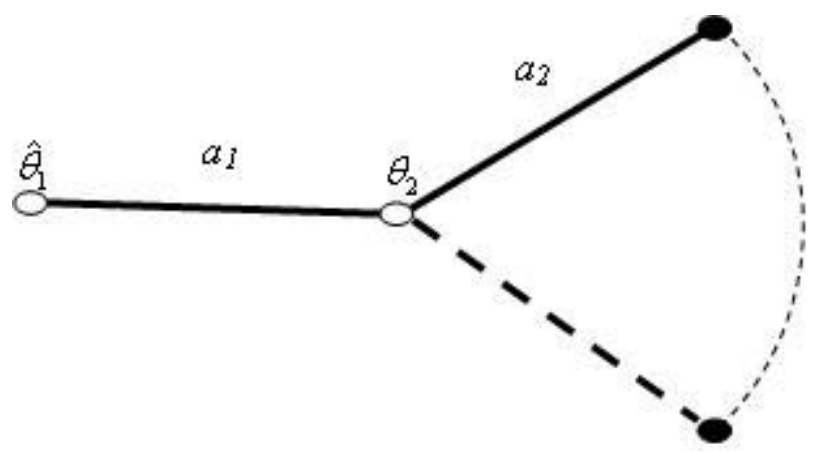

Figure 6: Aerial view of the manipulator with the first articulation blocked

The admissible region from the area of operation is, in this case, projected on a line of the arc as can be seen in figure 8 .

In this case, the terminal of the faulted manipulator can be placed in one of the two possible positions, A and A', from its trajectory. The kinematical constraints that can guarantee the existence of these two placement positions are defined like this: 


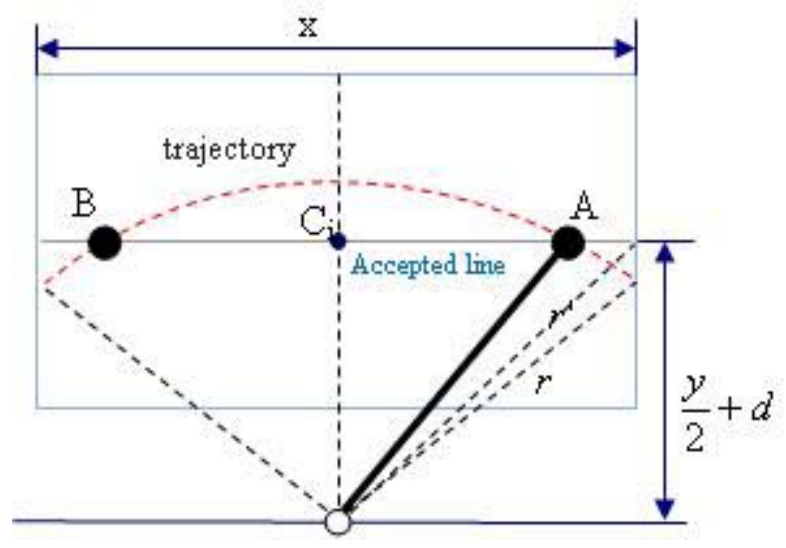

Figure 7: The kinematical constraints on case of first articulation failure

$$
\frac{y}{2}+d \leq r \leq r \prime
$$

Where $r$ is the radius of the radius of the arc and $r^{\prime}$ is the distance between the point in which the arm attaches itself to the support and the limits of terminal of the manipulator.

$$
\begin{aligned}
r \prime & =\frac{1}{2} \sqrt{x^{2}+(y+2 d)^{2}} \\
r & =a_{1} \cos \hat{\theta_{1}}+a_{2} \cos \theta_{2}
\end{aligned}
$$

where $\hat{\theta_{1}}$ is the angle of the jammed articulation. Note the fact that $r$ is identical to the length of the projection of the manipulator on the area of operation.

$$
\frac{y}{2}+d \leq a_{1} \cos \hat{\theta_{1}}+a_{2} \cos \theta_{2} \leq \frac{1}{2} \sqrt{x^{2}+(y+2 d)^{2}}
$$

\section{Dynamic Delay Compensation Modelled After Whale Commu- nication Habits}

This part of the paper deals with dynamic compensation of disturbances, [7] In the last decades, the amount of noise present in the ocean has increased significantly in the ocean. This is largely due to the increase of naval traffic, the exploitation of certain resources(e.g. oil through oil platforms) and the intensive use of sonars. The whales themselves have adapted by increasing the intensity of their sounds. With regard to robotic structure communication a dynamic delay compensation structure is necessary. Thus, alternative Smith predictor structures were used for the dynamic delay compensation, [2], [4], [5], [9], [8]. The following simulations were performed using Smith predictor structures on a robotic arm acted on by a Quanser SRV-02 servo-motor. 


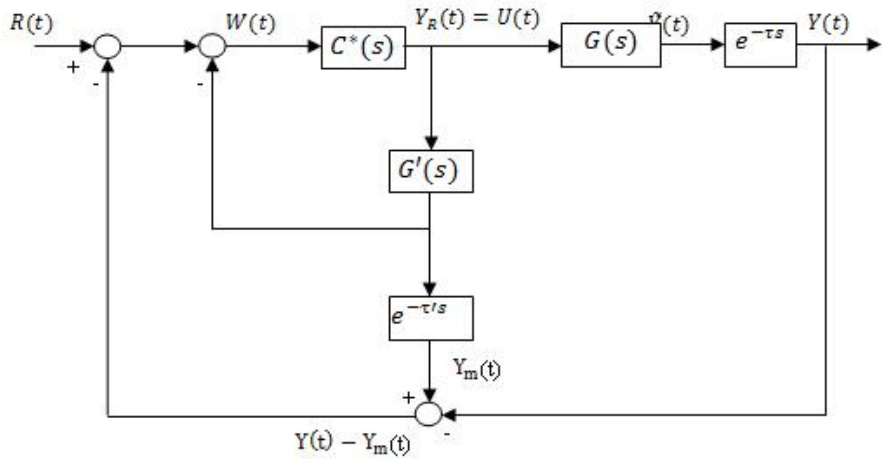

Figure 8: Modified Smith predictor structure

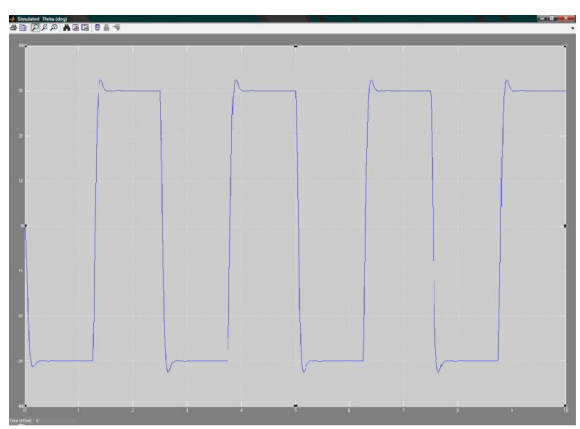

Figure 9: System response to a signal generator

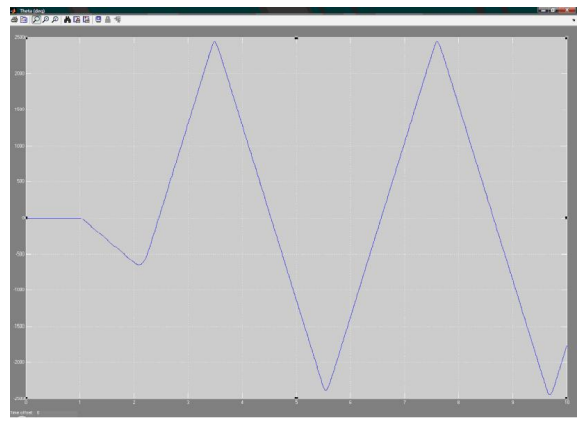

Figure 10: System response in case of delay occurence

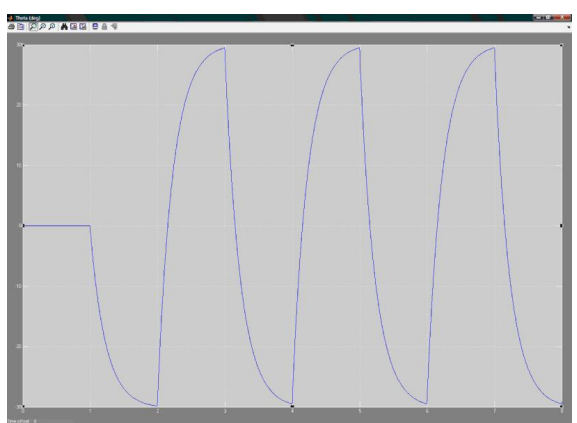

Figure 11: System response when using a modified Smith predictor 


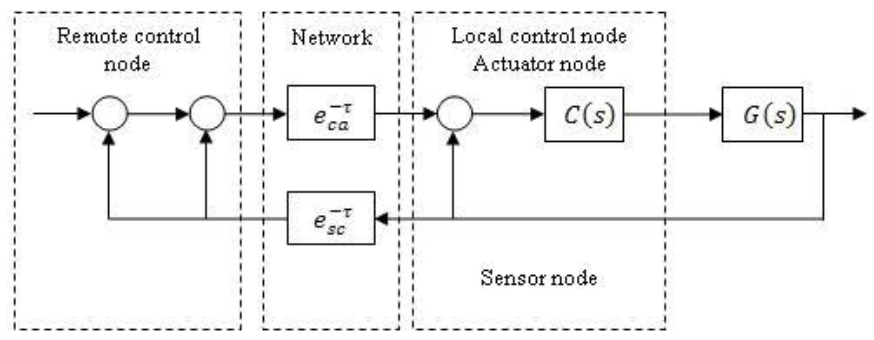

Figure 12: Improved Smith predictor structure

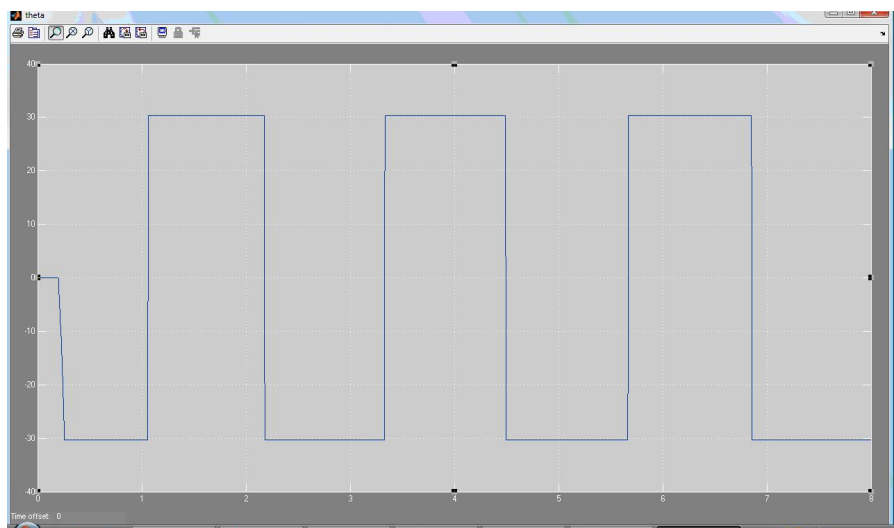

Figure 13: Position control of the Quanser SRV-02 plant using an improved Smith predictor

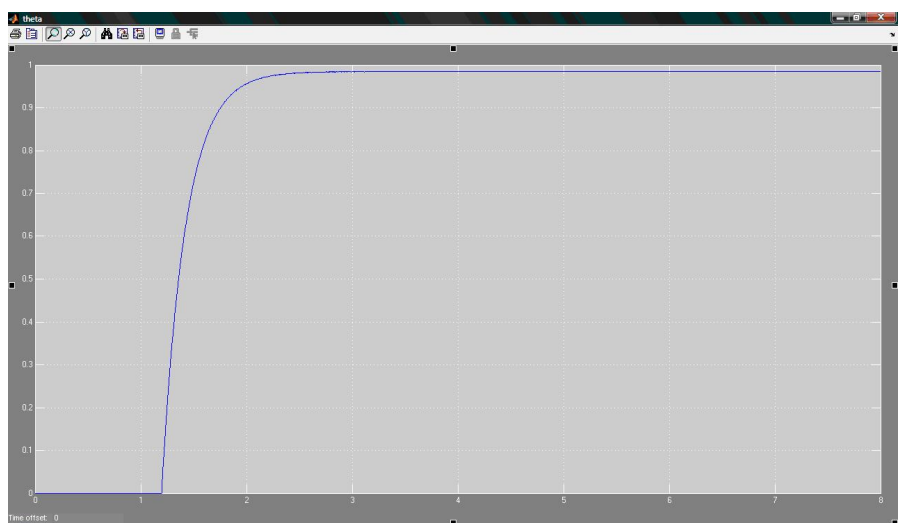

Figure 14: Step response of the system using the improved Smith predictor and a PI controller 


\section{Conclusions}

This paper analyses the possibility of modelling cooperative robot structures after the behavior and communication habits of whale pods. Certain key aspects are studied like the modelling of whale communication using data fusion techniques, determining the operation span in fault conditions and the dynamic delay compensation modelled also after whale communication. Aspects like communication and social structure of whale pods are well documented in literature. What this paper is trying to establish is that there is the possibility of implementing this complex behavior in a cooperative robot structure with a well determined purpose. Further research and the study of other key behavior aspects should lead to the appearance of another class of problems based on real-life phenomena-the evolution of whale pods in matters of communication.

Acknowledgement. This work was supported by the strategic grant POSDRU/89/1.5/S/61968, Project ID61968 (2009), co-financed by the European Social Fund within the Sectorial Operational Program Human Resources Development 2007-2013

\section{Bibliography}

[1] K.J. Astrom, B. Wittenmark, Computer Controlled Systems: Theory and Design, PrenticeHall, Englewoods Cliffs, NJ, 1984

[2] K.J. Astrom, C.C. Hang, B.C. Lin, A New Smith Predictor for Controlling a Process With an Integrator and Long Dead-Time, IEEE Transactions on Automatic Control, 39(2), pp. 343-345, 1994

[3] Y.U. Cao, A.S. Fukanaga, A.B. KAHNG, Cooperative Mobile Robotics: Antecedents and Directions, Autonomous Robots, 4, 1-23, Kluwer Academic Publishers, Boston, 1997

[4] D. Feng, D.,Wencai, L. Zhi, New Smith Predictor and Nonlinear Control for Networked Control Systems, Proceedings Of The International MultiConference of Engineers and Computer Scientists, March 18-20, Hong Kong, Vol. 2, pp. 1184-1188, 2009

[5] D. Feng, Q. Qian, Study of NCS with Improvement Smith Predictor and Fuzzy Immune Control, Proceedings of the International Conference on Intelligent Systems and Knowledge Engineering, Chengdu, China, 2007

[6] D.L. Hall, J. Llinas, Handbook of Multisensor Data Fusion, CRC Press, New York, USA, 2001

[7] J.G. Truxal, M.J. Shooman, W.R. Blesser, J.W. Clark, Remote Control, in the Handbook of Telemetry and Remote Control, Mc-Graw Hill, New-York, pp 1-169, 1967

[8] J. Velagic, Design of Smith-like Predictive Controller with Communication Delay Adaptation, World Academy of Science, Engineering and Technology, no. 47, pp 199-203, 2008

[9] M. Veronesi, Performance Improvement of Smith Predictor through Automatic Computation of Dead Time, Yokogawa Technical Report English Edition, pp 25-30, 2003

[10] X. Yang, Bio-Inspired Computing and Networking, CRC Press, 2011.

[11] http://www.eoearth.org/article/whale_communication_and_culture

[12] http://www.napali.com/na_pali_coast/hawaii_whale_watching/social_communication.html

[13] http://www.whales.org.za/facts_communication.aspx 